\title{
Formation of Electrical Synapses between Isolated, Cultured Helisoma Neurons Requires Mutual Neurite Elongation'
}

\author{
ROBERT D. HADLEY, ${ }^{2}$ DEANA A. BODNAR, AND STANLEY B. KATER ${ }^{3}$
}

Department of Biology, University of lowa, lowa City, lowa 52242

\begin{abstract}
Our previous experiments have suggested the hypothesis that conjoint active neuronal outgrowth may be necessary for formation of new electrical synapses between identified neurons of adult Helisoma buccal ganglia. This growth dependence hypothesis now has been tested by examining the responses of individual pairs of neurons in isolation from the influences of the ganglionic environment. Isolated cell culture of identified neurons (neuron 5) showed that: (i) neurons growing in cell culture undergo a predictable sequence of morphological changes culminating in a stable morphological state (i.e., growth stops); (ii) contact between actively growing neurons in cell culture results in the formation of electrical connections, just as in ganglia; and (iii) when an actively growing neuron encounters a neuron that is morphologically stable, electrical connections do not form or are very weak, even though strong connections are made between pairs of actively growing neurons in the same culture. These results establish that processes closely associated with growth are required for formation of electrical synapses between these neurons.
\end{abstract}

Electrical synapses exist in a great many tissues (e.g. Cox, 1974) and act as the substrate for both electrical and metabolic coupling between cells (Gilula et al., 1972; Pitts and Simms, 1977; Lawrence et al., 1978). Elcctrical synapscs arc an important subset of the kinds of connections that neurons make with one another (Bennett, 1977), and their strength can be accurately measured for quantitative comparisons (Bennett, 1966). Most importantly, for the present experiments, these connections easily can be induced to form between identified neurons under controlled conditions so that the phenomena affecting their formation can be studied.

In response to axotomy, buccal ganglion neurons of the snail, Helisoma, sprout and undergo a predictable sequence of changes in chemical and electrical connections (Murphy and Kater, 1978, 1980; Bulloch et al., 1980; Bulloch and Kater, 1981, 1982; Hadley et al., 1982, 1983; Hadley and Kater, 1983; Murphy et al., 1983).

Received August 29, 1984; Revised June 3, 1985;

Accepted June 4, 1985

${ }^{1}$ We wish to thank Paul Gade and Garry Hauser for technical assistance and Drs. J. Denburg and C. -F. Wu for cormenterts on the manuscript. This work was supported by United States Public Health Service Grants NS18819, NS15350, and HD18577.

2 Present address: Washington University School of Medicine, Department of Anatomy and Neurobiology, 660 South Euclid Avenue, St. Louis, MO 63110.

${ }^{3}$ To whom correspondence should be addressed.
These physiological changes all are associated with neuronal sprouting and outgrowth after axotomy.

The present experiments focused on the identified buccal ganglion neuron 5. The formation of a new electrical synapse between neuron 5 and the contralateral neuron 5 within the buccal ganglion entails two stages: first, neuron 5 connects with a specific group of neurons, and second, all but the 5-5 connection are selectively eliminated to yield the specific resultant connectivity pattern (Bulloch and Kater, 1981, 1982). Whereas this previous work (see also Bulloch et al., 1982) has examined factors affecting synapse elimination (cf. Dennis and Yip, 1978; Purves and Lichtman, 1980), the present communication examines constraints on the initial establishment of interconnected sets of neurons.

Previous studies (Hadley and Kater, 1982, 1983; Hadley et al., 1983) have suggested the hypothesis that active outgrowth from both neurons of a particular pair is necessary for formation of novel electrical connections. Such studies involved crushing nerves to axotomize neurons and evoke growth in order to examine the responses of single identified neurons. To remove possible ganglionic effects arising from such widespread axotomy, we have removed the yarnglion environment completely by maintaining the neurons 5 in isolated cell culture (Wong et al., 1981; Kater et al., 1982). This has enabled us to isolate the responses of the neurons from the effects of the environment and to demonstrate that the requirement for neuronal growth to form strong electrical synapses is an intrinsic property of these adult neurons.

A preliminary report of some of these results already has appeared (Hadley et al., 1983).

\section{Materials and Methods}

Animals from inbred stocks of Helisoma trivolvis were dissected (Kater and Kaneko, 1972) under sterile conditions (sterile antibiotic saline in a laminar flow hood; Wong ct al., 1981). Buccal ganglia were removed from the animals and prepared for neuronal isolation for cell culture.

Helisoma physiological saline contained (in millimolar concentration): $\mathrm{NaCl}$ $51.3 ; \mathrm{KCl}, 1.7 ; \mathrm{CaCl}_{2}, 4.1 ; \mathrm{MgCl}_{2}, 1.5 ; \mathrm{HEPES}, 5$, al $\mathrm{pH}$ 7.3. Culture medium was modified Leibowitz L-15 for Helisoma (HL-15; Wong et al., 1981). Neurite outgrowth was dependent upon the presence of a conditioning factor(s) from Helisoma CNS (Wong et al., 1981, 1983a, b; Barker et al., 1982). Consequently, isolated neurons were cultured in $\mathrm{HL}-15$ conditioned medium (CM; 2 brains/ml for $72 \mathrm{hr}$ ) to allow outgrowth to occur.

For isolation of neuron 5 , buccal ganglia were treated with $0.2 \%$ trypsin (Sigma type III in sterile saline) for $1 / 2 \mathrm{hr}$ followed by a 15 -min rinse in $2 \%$ trypsin inhibitor (Sigma type I-S). Ganglia were then pinned to Sylgard pads in HL-15 medium (Wong et al., 1981). Identified neurons 5 were removed by making an incision in the ganglionic sheath above neuron 5 with a tungsten microknifc, exposing the neuron. The neuron was removed with a micropipette controlled by micromanipulation and a micrometer drive syringe (see also Kater et al., 1982). Neurons were placed in polylysine-coated culture disties cornlaining $\mathrm{CM}$. Neurons initially plated on day 0 were designated primary neurons, and subsequent cells plated in the same culture dish were 
designated secondary neurons. Procedures for removal and plating of secondary neurons were the same as for primary neurons. Addition of secondary neurons was at either day 2 or day 7 . Cultures were maintained in sterile plastic cabinets with humidified air at 22 to $25^{\circ} \mathrm{C}$.

Electrophysiology and photography. Standard electrophysiological and morphological techniques were used. Glass, fiber-filled microelectrodes were filled with $1.5 \mathrm{M} \mathrm{KCl}$ (DC resistance $=20$ to 60 megohms) and connected through a unity gain, high input impedance amplifier to an oscilloscope and a chart recorder. Electrical coupling was measured by injecting current into one neuron through a Wheatstone bridge circuit and recording the coupling coefficient (postsynaptic/presynaptic voltage ratio; Bennett, 1966). Electrical coupling was assayed 24 to $48 \mathrm{hr}$ after initial morphological contact. Physiological recordings and photography of isolated neurons were performed on an inverted microscope with phase contrast optics.

Comparisons between groups of data were made using the Student's $t$ test with a correction for unmatched variance (Dixon and Massey, 1969). Values for electrical coupling within experimental groups are expressed as mean \pm SEM. Curve-fitting in Figure 11 was done by performing a linear regression on the log of coupling coefficient versus distance. A curve was then constructed by eye through representative regression points along the linear axis.

\section{Results}

Growth kinetics of neuron 5 in cell culture. Initial cell culture experiments determined the kinetics of neurite outgrowth for neurons 5 and then related those kinetics with the morphology of the growth cones. Neurons plated in Helisoma brain CM (see Wong et al., 1981; Kater et al., 1982), expressed a reproducible sequence of morphological changes. Isolated spherical cell bodies attached to the substratum (Fig. 1A) and initiated neurite sprouting within 1 day. These neurites were extended behind large, flat, phase-dark growth cones (Fig. 1B), with actively changing shapes. During this stage of growth, rates of extension for individual neurites were relatively constant (Fig. 2, top): approximately $300 \mu \mathrm{m} /$ day between days 1.5 and 3 (at 22 to $25^{\circ} \mathrm{C}$ ). After the active growth stage, neurites underwent a morphological transition; growth cones became quiescent, slender, and phase bright (Flg. $1 \mathrm{C}$ ), and neurite extension slowed and stopped (Fig. 2, top; after day 3). After this stage, further neurite growth was not observed, although small adjustments could have gone undetected. The time course shown in Figure 2, top, was derived from observations on five individual neurites of two neurons and exemplifies the transition to a stable state ( \pm 1 day) made by all neurons 5 plated in $\mathrm{CM}$. Resting and action potential amplitudes of stable neurons were indistinguishable from those of actively growing neurons. Attainment of similar stable morphological states has been described previously for isolated, unidentified Helisoma neurons, and is a predictable feature for all neurons we have observed in culture (Wong et al., 1981; Kater et al., 1982).

The morphology of individual growth cones was a reliable indicator of the growth status of the neurons (Flg. 1, insets). Slender, phasebright growth cones were characteristic of morphologically stable neurites (Fig. 1C). Examination of all terminal structures on a population of neurons 5 (Fig. 2, bottom) showed that the proportion of phase-bright endings increased rapidly with time, such that by day $3,83 \%$ of the endings were phase bright. The time course of increase of phase-bright endings was coincident with the cessation of neurite extension. These distinct morphological correlates to growth allowed quick and reliable classification of individual neurons and tests for competence to make electrical synapses as a function of growth status.

It was important to confirm that stable-state morphology was not the result of changes in the culture conditions with time. Figure 3 shows a pair of stable-state neurons after 4 days in culture, to which was subsequently added a third neuron (Fig. 3, arrow) to test the ability of the medium to support outgrowth. This neuron grew rapidly and likewise reached a stable state indistinguishable from that seen in fresh medium. Thus, the attainment of stable state was not a result of changes in the $\mathrm{CM}$ or conditioning factors.

Formation of electrical connections between simultaneously plated neurons. As many as 12 neurons were cultured simultaneously in a single culture dish in $\mathrm{CM}$, and growth was essentially synchronous for all of the neurons (but see Haydon et al., 1984, for
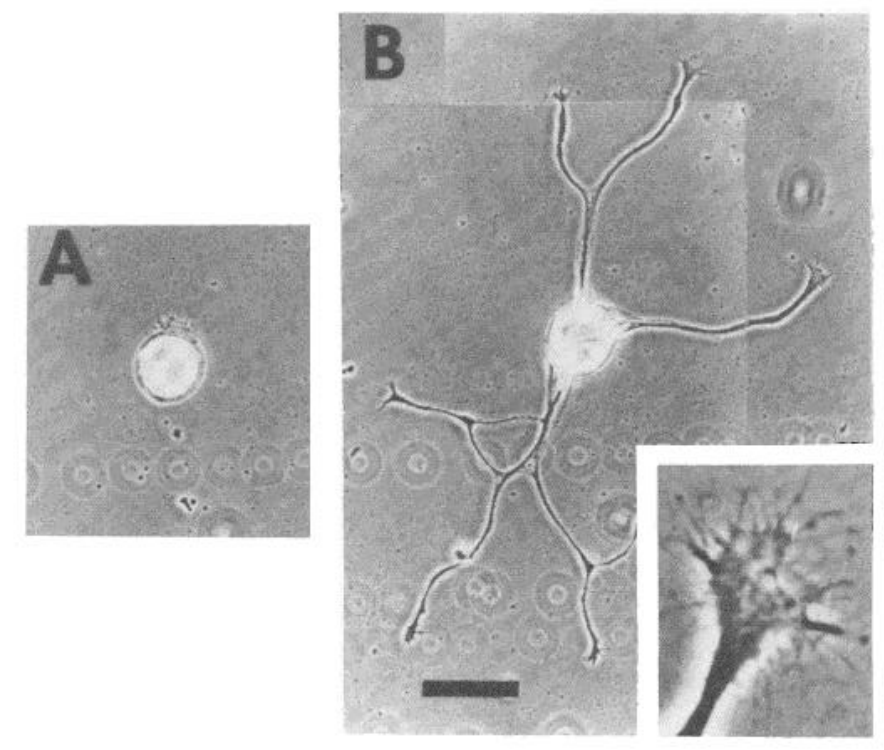

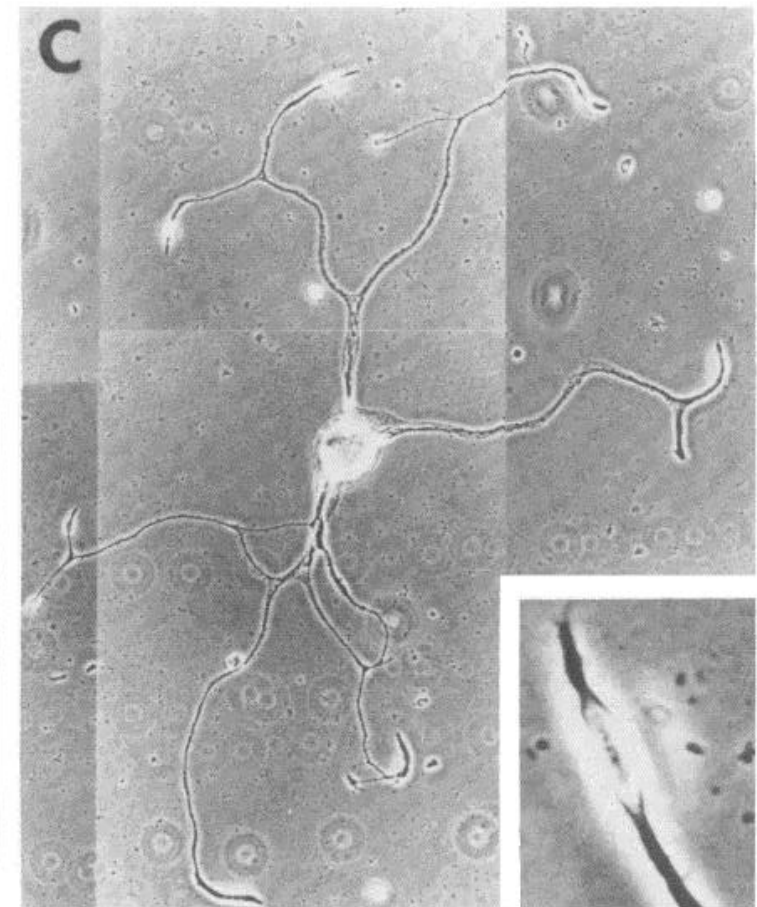

Figure 1. Stages in the growth of an isolated neuron 5. A, Initiation of outgrowth occurs with the evolution of veils around the spherical cell body, usually within $24 \mathrm{hr}$ of initial plating. $B$. Active growth occurs after initiation of sprouting. Growth cones (insets) are characteristically large, flat, and phase dark during active growth. C, A stable morphology is reached after which no more growth ensues. The stable state is characterized by slender, phase-bright endings. $B a r=100 \mu \mathrm{m}$ for $A$ to $C ; 20 \mu \mathrm{m}$ for insets. 

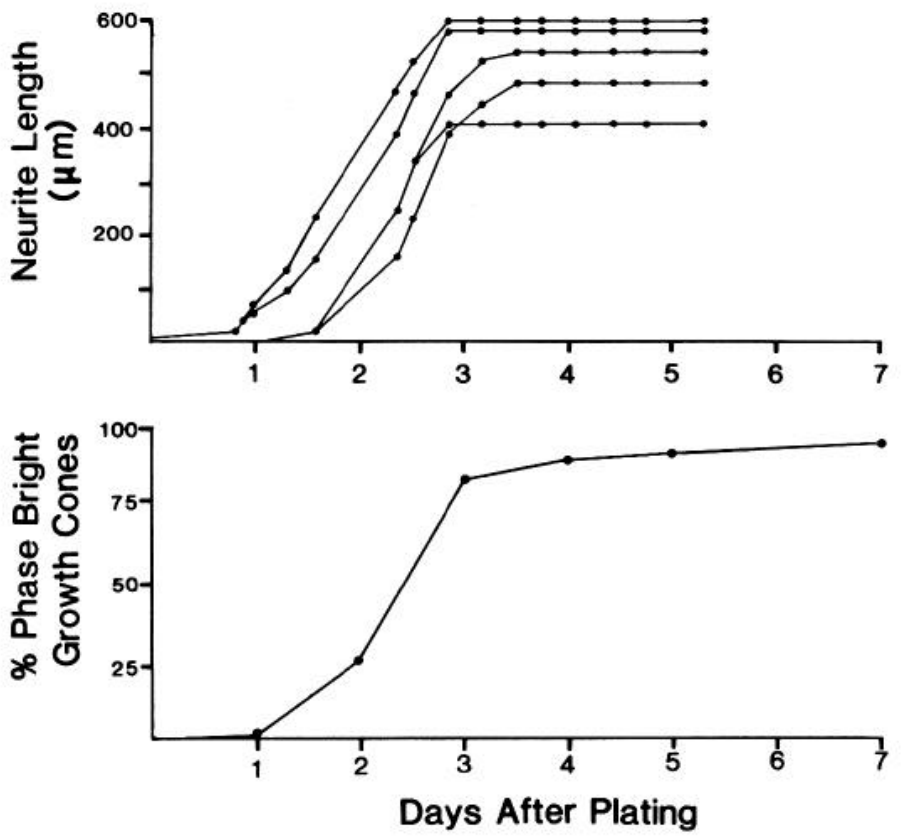

Figure 2. Quantitative measures of transition from active growth to stable morphology. Top, Lengths of individual neurites were measured at intervals after initial plating of neuron 5 . The slope of the graph indicates growth rates. A sequence of initiation, rapid growth, and transition to a nongrowing state is apparent from this analysis of 5 individual neurites from two neurons. Bottom, The percentage of endings which were phase bright was determined from photographs of 11 neurons at different times after initial plating. This population of endings ( $n=89$ to 175 for each point) shows an increase to nearly $100 \%$ phase-bright by day 7 .

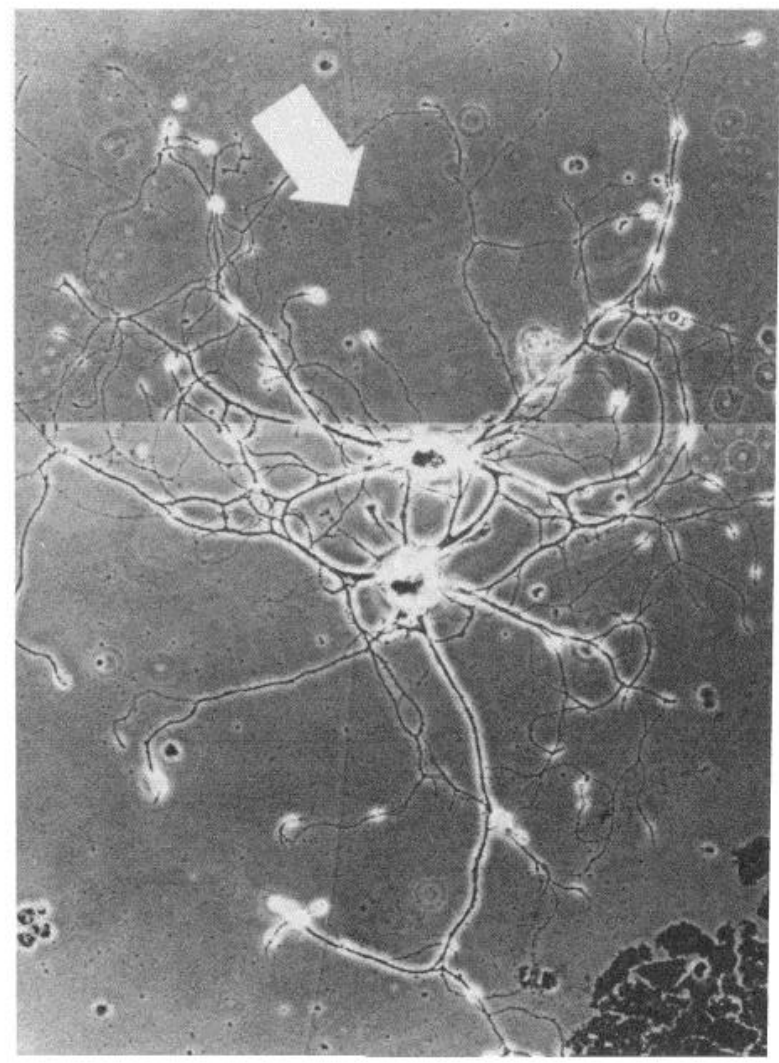

different results using different culture conditions). Contact invariably occurred between the growing neurites of neurons plated within 500 $\mu \mathrm{m}$ of one another (Fig. 4). Pairs of such "primary" neurons (i.e., those neurons plated on day 0 ) which were assayed by intracellular recordings 24 to $48 \mathrm{hr}$ after contact always were electrically coupled to one another. Current injected into one neuron caused a voltage deflection in the coupled neuron (Fig. 4, insets), and action potentials in one cell could give rise to action potentials in the other cell or to electrotonic postsynaptic potentials if coupling was weak and/or if the postsynaptic cell were slightly hyperpolarized by injected current. In every case, coupling was nonrectifying (i.e., current could be passed in both directions) (Fig. 4 , insets $A, B$, and $C$ ). The mean coupling coefficient for neuron pairs assayed 24 to $48 \mathrm{hr}$ after initial contact was $0.42 \pm 0.10(n=9)$. The electrical coupling in cell culture was essentially identical to that observed between these neurons in the ganglion.

When neurons were part of a simultaneously plated multicellular network, coupling was observed between all pairs of neurons that were touching one another. Coupling was measured by pairwise recordings and, therefore, values included direct as well as indirect electrical pathways via other neurons. All ( $n=30$ pairs) simultaneously growing pairs of neurons that we assayed became electrically coupled. A single neuron was capable of forming electrical connections with more than one homologue; this observation is unique to cell culture and could not have occurred in organ culture within the buccal ganglion environment (see Bulloch and Kater, 1981, 1982; Hadley et al., 1982; Hadley and Kater, 1983).

Differential competence of growing or stable primary neurons for electrical synapse formation. Cell culture not only allowed multiple neurons to connect, but also permitted delayed addition of "secondary" neurons to the previously plated primary neurons. Neurons were added near the primary neurons either on day 2 , when the primary

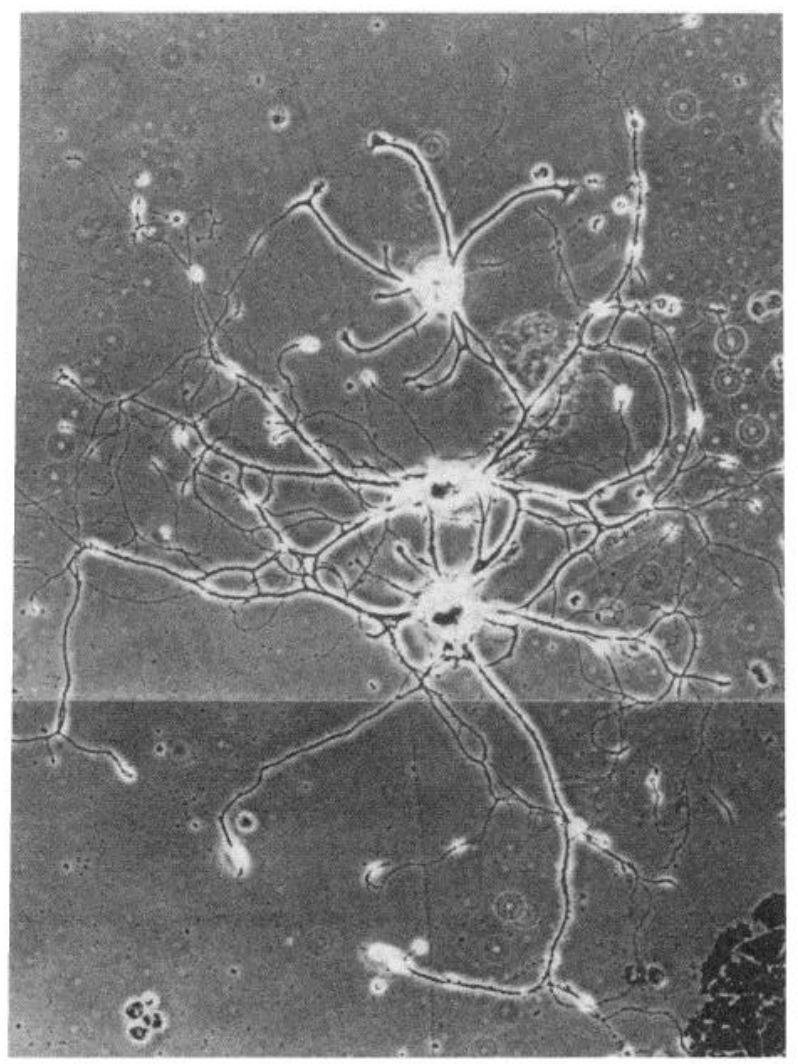

Figure 3. Supportive culture conditions after neurons have reached stable states. After 4 days, when two neurons 5 had reached stable morphologies (left), a third neuron 5 was added to the culture (at arrow). The added neuron grew indistinguishably and also reached a stable state within 3 days, indicating no change in the culture environment. 


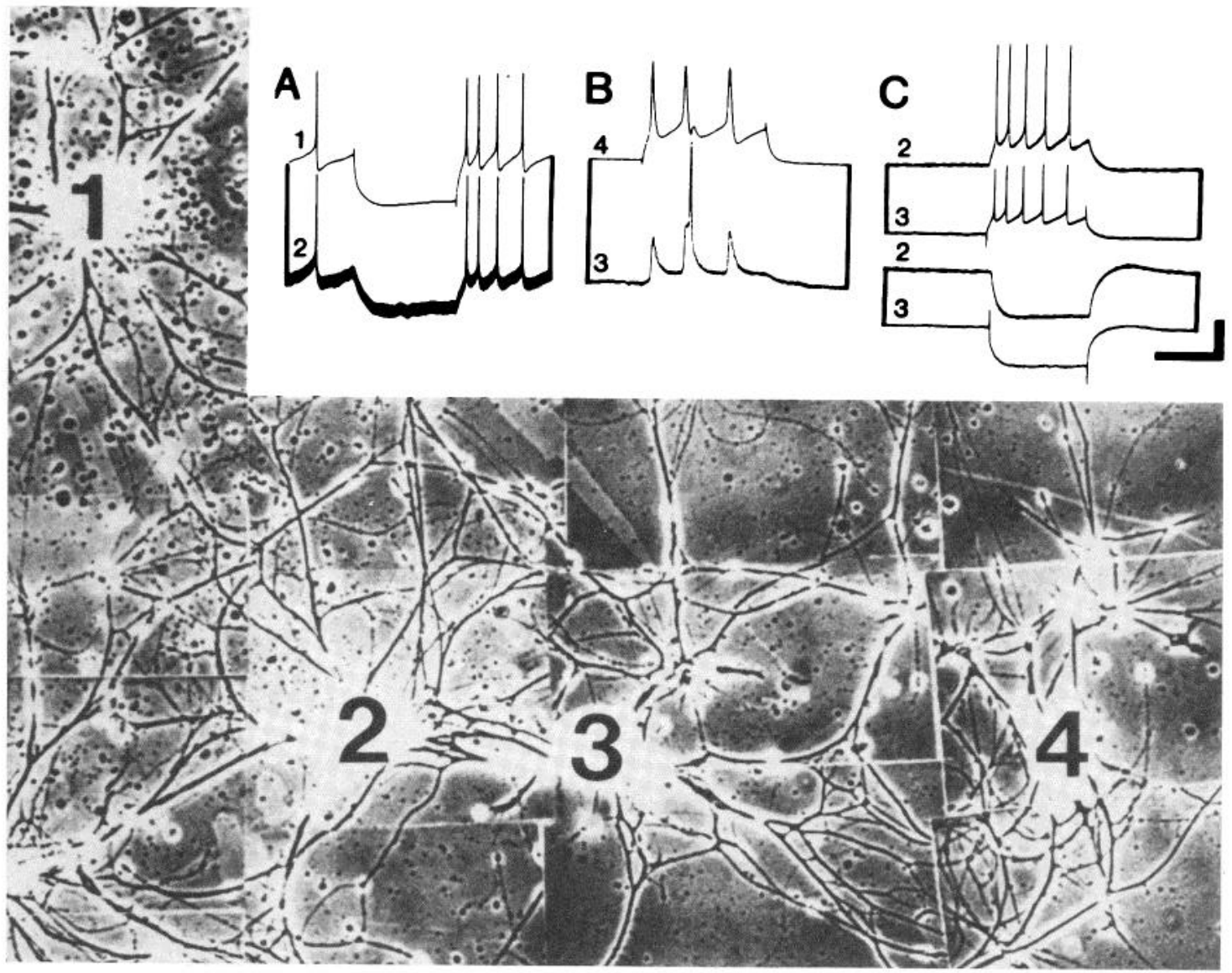

Figure 4. Morphology and electrical interaction among four simultaneously plated neurons. Neurons were photographed $48 \mathrm{hr}$ after isolation from the buccal ganglion and initial plating near one another. Intracellular recordings from these neurons (traces identified by numbers 1 to 4 ) are shown in the insets. A, All neurons were DC electrically coupled (current injected in upper trace) and spontaneous action potentials often propagated with fidelity. $B$, With neuron 3 held slightly hyperpolarized, action potentials driven in neuron 4 by a depolarizing current pulse gave rise to electrotonic postsynaptic potentials in neuron 3 . Note also the electrotonic potential in neuron 4 rising from the single action potential in neuron 3 . C, Electrical coupling was bidirectional and nonrectifying. DC current (injected into neuron 3 ) passed in both positive and negative polarities. Calibrations: $10 \mathrm{mV}$ for all; $2 \mathrm{sec}, A$ and $C ; 0.5 \mathrm{sec}, B$.

neurons were actively growing (Fig. 5), or on day 7, when they were in a stable morphological state (Fig. 6). Secondary neurons grew indistinguishably from the originally plated neurons, and primary/ secondary neuron contact (at the level of the light microscope) occurred within 1 to 1.5 days after plating of secondary neurons. Electrical coupling was assessed 24 to $48 \mathrm{hr}$ after primary/secondary neuron contact (Figs. 7 and 8 ). Typically, recordings from the resulting neuronal networks showed strong coupling between the initially plated neurons as well as between the pairs of secondary neurons. Strong primary/secondary coupling occurred only if primary neurons were actively growing and, conversely, negligible coupling occurred if primary neurons were stable. Thus, in agreement with previous experiments in buccal ganglia (Hadley and Kater, 1983), the stable morphological state of these neurons correlated with a lack of competence to make new electrical connections.

Quantitative comparisons of coupling between neuron pairs were made using group mean coupling values (Fig. 9). Coupling between stable primary and secondary neurons (SP/S) was strikingly lower than any of the other groups (primary/primary $=P / P$, growing primary $/$ secondary $=\mathrm{gP} / \mathrm{S}$, secondary $/$ secondary $=\mathrm{S} / \mathrm{S}$ ). Moreover, the occurrence of zero coupling values for SP/S (7 of 15 with undetectable coupling) was significantly more frequent than in the other groups with conjoint growth $(0$ of 33 with no coupling, $p<$ 0.001 by contingency $\chi^{2}$ test). Furthermore, $S / S$ readily connected to one another, with significantly higher coupling coefticients compared with $\mathrm{SP} / \mathrm{S}$ ( $p<0.005$; see also "Discussion" for somewhat lower values of $\mathrm{S} / \mathrm{S}$, compared to $\mathrm{P} / \mathrm{P}$ and $\mathrm{gP} / \mathrm{S}$ ).
These comparisons demonstrate a clear difference in the synaptogenic competence of growing and nongrowing neurons in the same culture dish.

Additional influences on electrical coupling. A number of phenomena other than growth status also might be considered as possible bases for the lack of coupling between stable primary neurons and growing secondary neurons, including the following.

(i) The culture medium was 7 days old at the time of addition of secondary neurons, and its quality might have deteriorated during this time. Experimental results, however, mitigate against this possibility: after plating on day 7 , secondary neurons sprouted and grew identically to primary neurons plated on day 0 , indicating continued action of growth-promoting factors in the medium (Figs. 3 and 6; see Barker et al., 1982, and Wong et al., 1983a, b, for characteristics of the growth-promoting factors). The secondary neurons also had normal electrophysiological characteristics and readily coupled with one another (Figs. 8 and 9), confirming that the medium was not greatly changed.

(ii) It is possible that intercellular affinities (e.g., adhesion) may have differed between growing and nongrowing neurons. We have not directly measured adhesive strengths (e.g., Bray, 1979); however, growing secondary neurites appeared to "track" and follow both growing and nongrowing neurites equally well. This would indicate that the nongrowing cell surfaces were still "preferred" to the polylysine substratum by growth cones, and adhesion probably was not significantly reduced.

(iii) If the amount of neurite contact could affect coupling strength, 

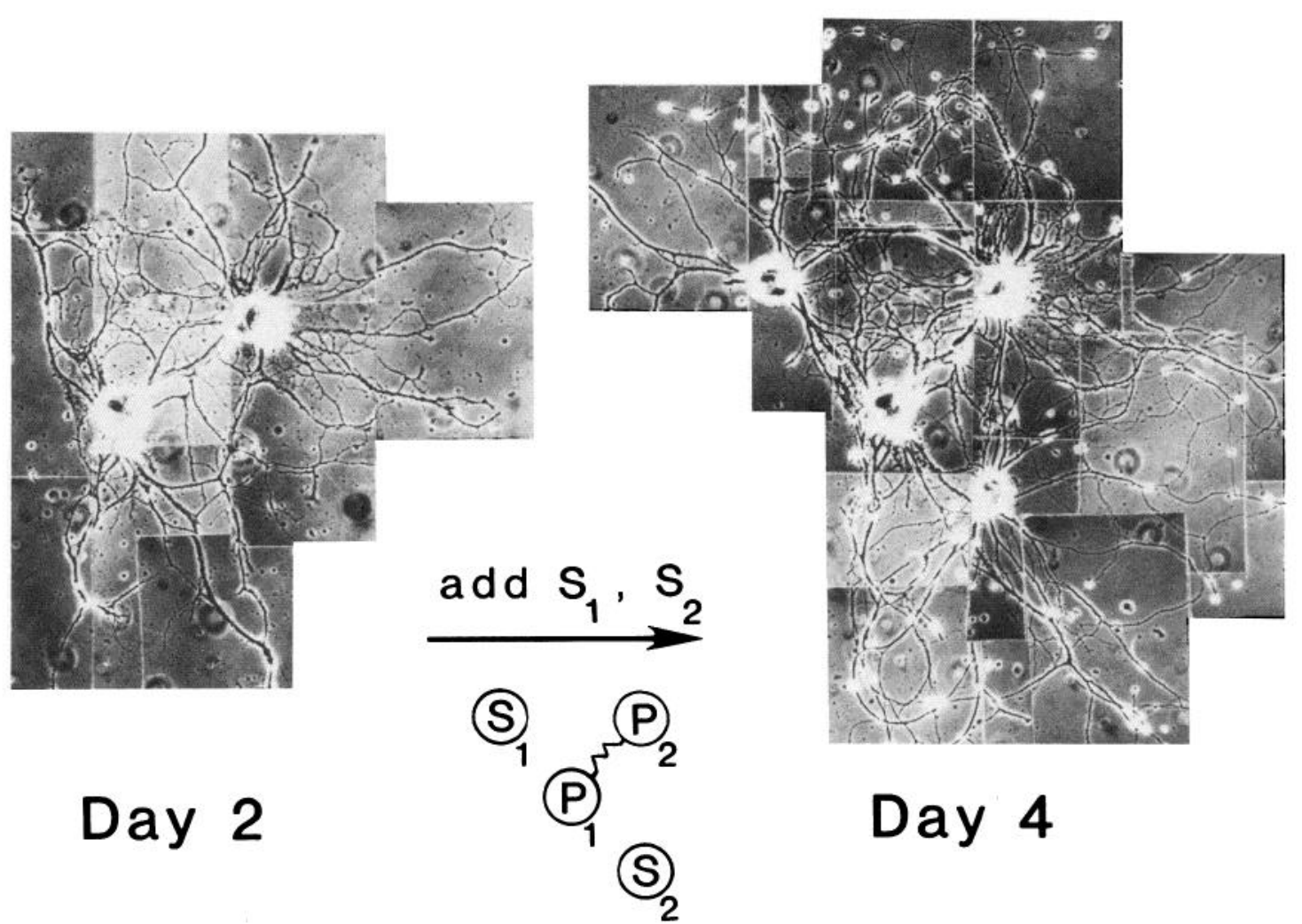

\section{2}

Figure 5. Addition of secondary neurons to cultures of growing primary neurons. Two primary neurons $(P 1, P 2)$ were allowed to grow and interact until day 2. Two secondary neurons $(S 1, S 2)$ were added after the time of the left photograph. Sprouting and growth ensued from the added neurons in a manner indistinguishable from that expressed by primary neurons. By day 4, growth from the added neurons overlapped the earlier growth. The primary neurons were still undergoing active growth at the time the secondary neurons were added (see the text and Fig. 7).
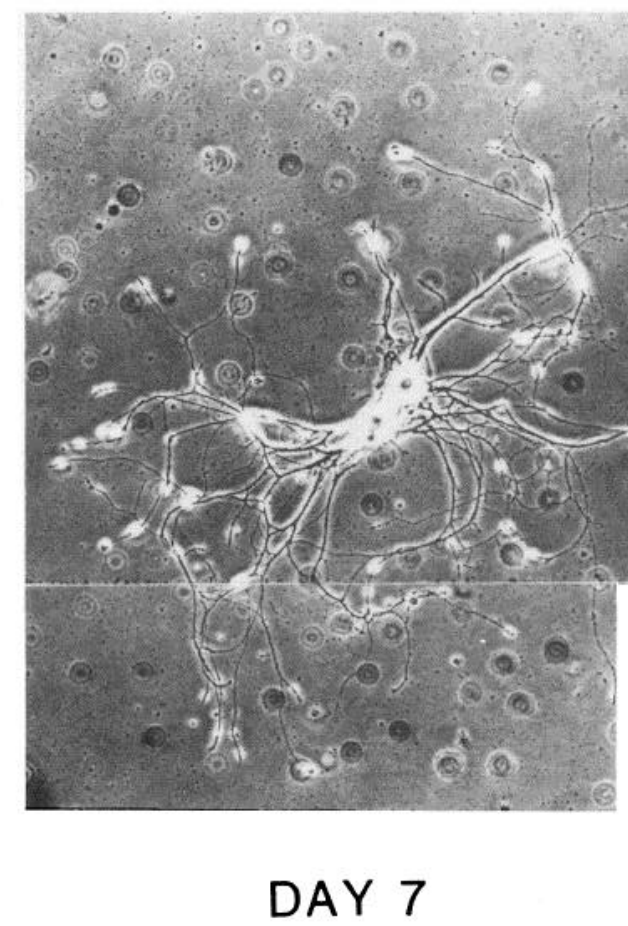

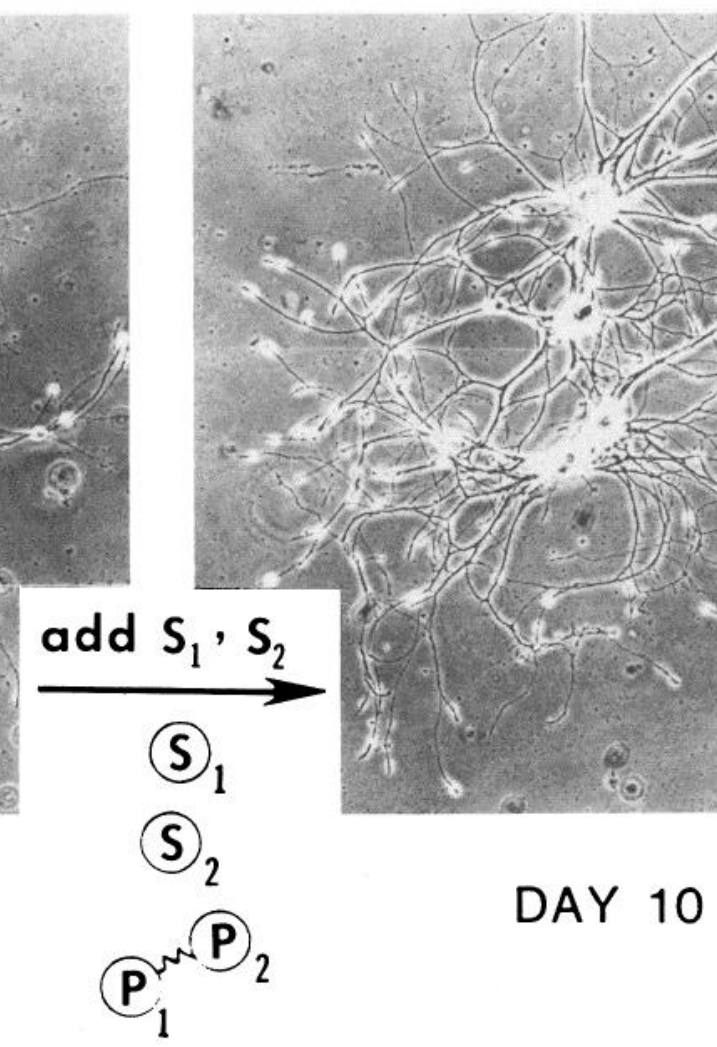

Figure 6. Addition of secondary neurons to stable primary neurons. These primary neurons $\left(P_{1}\right.$ and $\left.P_{2}\right)$ were stable on day 7 (note phase-bright endings). Secondary neurons $\left(S_{1}\right.$ and $\left.S_{2}\right)$ which were added on day 7 (after the time of the photograph on the left), grew and contacted the primary neurons by day 10. 

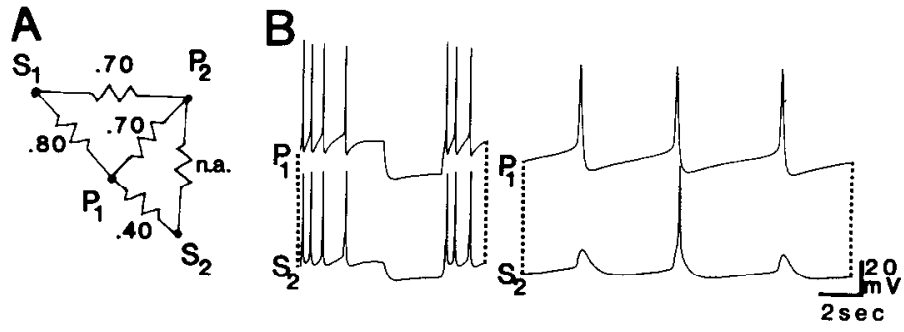

Figure 7. Electrical coupling between actively growing primary neurons and added secondary neurons. $A$, The network of electrical coupling for the neurons shown in Figure 5 shows that strong primary/secondary coupling was readily established. Some connections were not assayed (n.a.). Representative coupling traces from the network show that action potentials and direct current are transmitted even between a pair of cells with relatively low coupling (P1-S2; $\left.V_{2} / V_{1}=0.40\right)$. $B$, At left, current injected into $P 1$ is seen in S2 and action potentials are faithfully transmitted to S2. At right, with a slight hyperpolarizing current injected into S2, action potentials in P1 give rise to both electrotonic postsynaptic potentials and action potentials. Calibrations: $20 \mathrm{mV}$ for all; $2 \mathrm{sec}$, left; $0.5 \mathrm{sec}$, right.
A

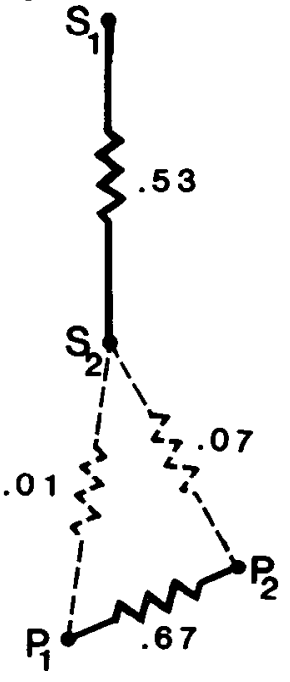

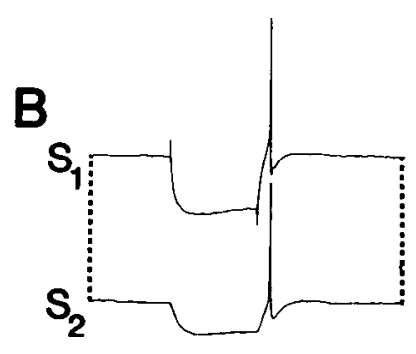

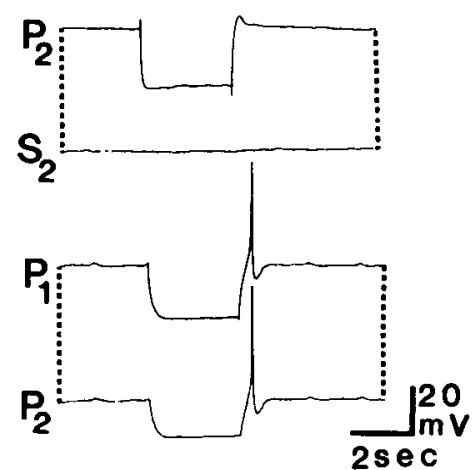

Figurc 8. Electrical connections among primary and secondary neurons shown in Figure 6 . A, Summary of the coupling networks shows strong $P / P$ and S/S coupling, whereas primary/secondary coupling (dashed resistors) was very weak. $B$, Representative coupling records from these neurons (all traces at the same gain) show the relative physiological effects of this strong and weak coupling. Current was injected into the neurons in the top traces.

then it is possible that secondary neurons could have simply experienced little neurite overlap with stable primary neurons. In general, however, the physical overlap between coupled pairs and noncoupled pairs of neurons was similar. Figure 10 shows that even when there was considerably more overlap between a secondary and a stable primary neuron, coupling was much weaker than between primary neuron pairs with less overlap. Differences in amount of neurite contact therefore did not account for the observed coupling differences.

(iv) Another possibility is that prior stabilization of $\mathrm{P} / \mathrm{P}$ connections could confer a "resistance" to incoming new connections (e.g., Slack, 1978; Thompson, 1978; Bennett et al., 1979). Two classes of observations indicate that this was not the case. First, in two experiments, when we plated solitary primary neurons and allowed them to reach a stable morphological state without connecting to other neurons, secondary neurons did not connect with them (cou- pling coefficients $=0$ and 0.01 ). Additionally, the prior formation of connections did not prevent connections between growing primary and secondary neurons (Fig. 9, gP/S). The formation of prior P/P connections is thus neither necessary nor sufficient to prevent neuron pairs from connecting. The simplest hypothesis is still preferred: that growth, or a cellular function intimately related to growth, is necessary for the formation of electrical synapses between these neurons.

In addition to growth, we have identified one other factor that affected the strength of coupling. Coupling coefficients between neurons depended upon intervening distances as well as growth status. A plot of coupling versus distance (Fig. 11) shows an inverse distance-coupling relationship for growing pairs of neurons, whereas secondary to stable primary coupling (Fig. 11, open circles) is low or nonexistent at all distances. Coupling between neurons was expected to be reduced with greater distances between somata (recording sites) both from electrotonic decay and fewer opportunities for neurite contact (assuming radial outgrowth). A simple model of electrotonic decay would predict a logarithmic relationship between coupling and distance. A least squares regression using this model (line plotted in Fig. 11) yielded a correlation coefficient $(r)$ of -0.79 , indicating a possible contribution of electrotonic decay in this relationship. Other factors, such as decreasing junctional contact, almost certainly also contributed to the lower coupling with distance.

\section{Discussion}

We have addressed the question of the requirement of neurite outgrowth for electrical synapse formation in a variety of experimental paradigms. With growth evoked in intact ganglia by selective nerve crushes, pairs of growing neurons always connected, whereas when growth was evoked from only a single neuron of a pair, the result was very weak or absent electrical connections (Hadley and Kater, 1983; Hadley et al., 1983). Spatially coincident growth was thus

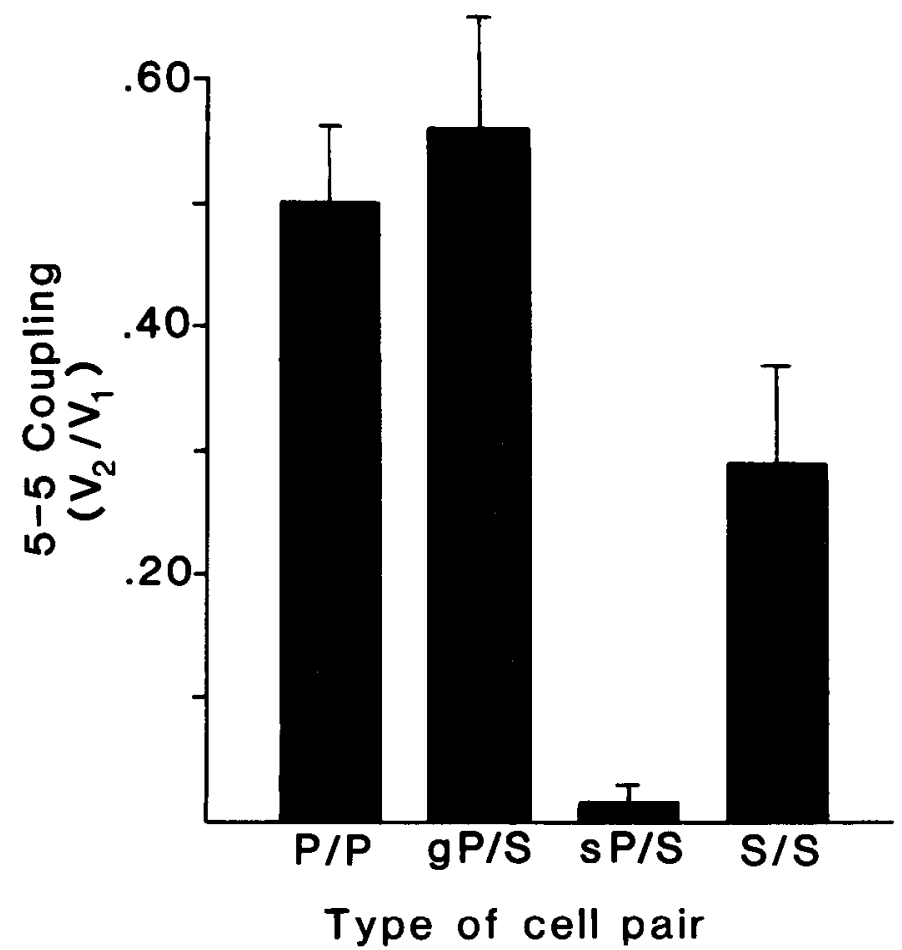

Figure 9. Summary of mean coupling coefficients for all groups. Comparisons of primary/primary $(P / P)$ and growing primary/secondary $(g P / S)$ show no difference. Secondary/secondary $(S / S)$ values are somewhat lower than $\mathrm{P} / \mathrm{P}$ and $\mathrm{gP} / \mathrm{S}(\rho<0.1$ and $\rho<0.05$, respectively). Coupling between secondary and stable primary neurons $(S P / S)$ is significantly lower than any of the other groups $(p<0.005)$. Significance levels are from paired $t$ tests. Values are mean \pm SEM, $n=7$ to 17 for each group. 


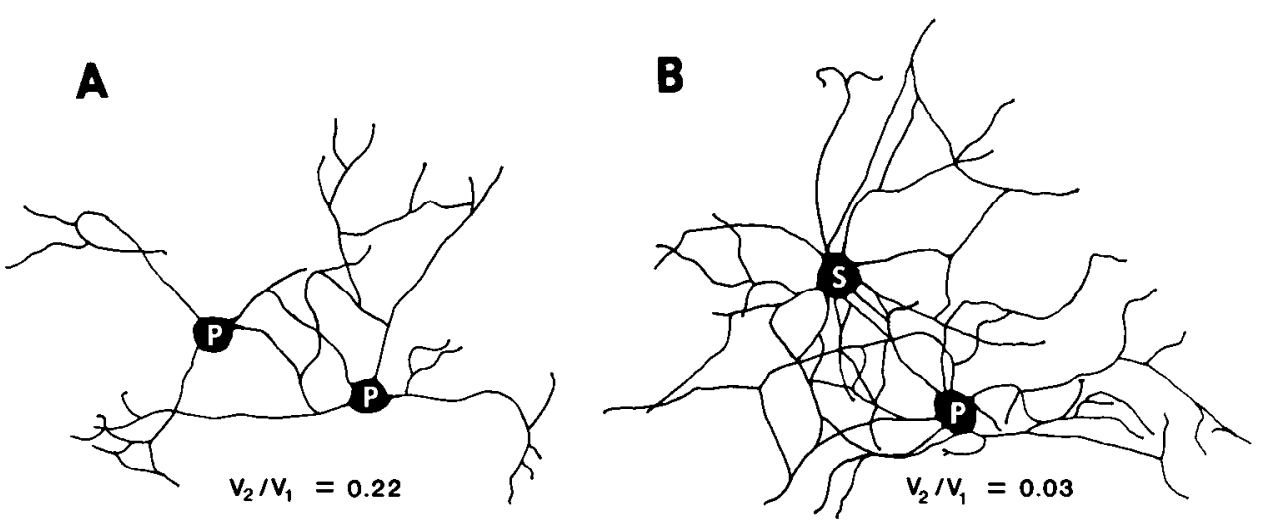

Figure 10. Neurite overlap for conjointly growing $(A)$ and growing/stable $(B)$ pairs of neurons 5. Although there was abundant physical overlap between a secondary neuron and stable primary neuron ( $S$ and $P$ ), coupling was much lower than between two growing primary neurons with less contact between them $(P$ and $P)$. These tracings were taken from photographs with multiple other neusons in the field. All primary neurons were plated on day 0 , secondary neurons on day 7 . Coupling measurements were performed on day $2(A)$ or day $9(B)$.

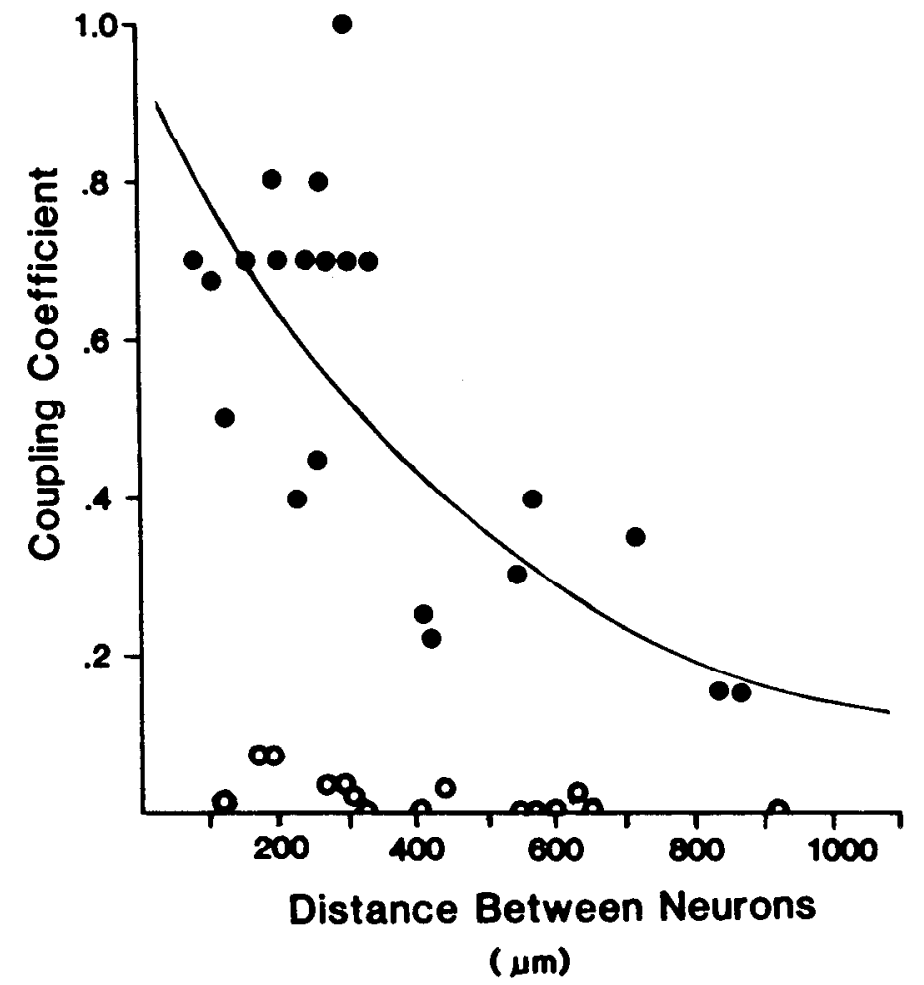

Figure 11. Scatter plot of coupling as a function of distance between neurons. Each point represents a single neuron pair. Solid circles are P/P and $\mathrm{gP} / \mathrm{S}$, and the line is the least squares logarithmic regression plot for those points. Open circles are sP/S and half circles represent sP/S coupling values of zero. Distances were calculated from photographic negatives.

required in situ. Growing peripheral axons of neurons $5 \mathrm{R}$ and $5 \mathrm{~L}$ also can couple to one another, making an electrical synapse in the periphery (Hadley et al., 1982), showing that the central ganglionic environment was not required, and spatially coincident growth was sufficient for connections to occur. Finally, in cell culture (this communication), isolated pairs of simultaneously growing neurons always connected, in contrast with the failure of stable neurons to form electrical synapses. Thus, temporal coordination, as well as spatial coordination of growth, is necessary for formation of electrical synapses, both in situ and in vitro.

It seems likely that the physical substrate for electrical coupling is the presence of gap junctions between these neurons. D. Hall (personal communication; Hall et al., 1984) has demonstrated gap junctions by freeze fracture in the buccal ganglion and in thin seclions of olher Helisoma neurons in cell cullure.

Coupling in cell culture (as in situ) occurred not only between neurons 5. but also between homologous pairs of identified neurons
4 or 19, and between heterologous pairs (e.g., 5-19; Cohan and Kater, 1983). All of these pairs were plated simultaneously, so that growth dependence for the resulting electrical connections has not been evaluated. It will also be of interest to define the conditions necessary for selective elimination of some connections (e.g., 5-19 or 5-4) and maintenance of others (e.g., 5-5) in cell culture, as occurs in ganglionic organ culture (Bulloch and Kater, 1982; Hadley and Kater, 1983).

The use of isolated cell culture represents an attempt to minimize the influence of surrounding nervous tissue in order to define the intrinsic properties of neurons that might affect connectivity. In earlier experiments which employed nerve crushes for axotomy, competence of neurons to make electrical synapses was presumably intrinsic to growing neurons but could also have been strongly influenced by the ganglionic environment and nearby populations of other axotomized neurons. In a number of systems, trans-synaptic effects (e.g., Sumner, 1975, 1977; Cotman, 1978), or diffusible substances from other damaged neurons (Longo et al., 1981, 1982; Nieto-Sampedro et al., 1982), or target tissues (Brown et al., 1981) can induce or enhance neuronal sprouting. Similar phenomena do occur in Helisoma: buccal ganglion neurons 19R and 19L sprout and grow after nerve trunks which do not contain their axons are crushed, even when connections with their targets are intact (Murphy, 1979). The similarity of results in whole ganglia (Hadley and Kater, 1983; Hadley et al., 1983) to the present results in cell culture, however, support our growth dependence hypothesis and indicate a minimal role of nerve crush damage.

In order to use morphologically stabilized neurons as models for a nongrowing state, the culture conditions must remain supportive. It is important to note that stable primary neurons appeared to be as viable as actively growing ones. For example, no significant differences were seen for resting or action potential amplitudes of primary neurons at day 9-10, compared with day 2-3. Additionally, profuse outgrowth of secondary neurons added to 7-day cultures showed that there was no decrease in the ability of CM to support growth cone formation and neurite elongation. Coupling between pairs of secondary neurons also indicated continued support for establishment of electrical synapses.

The lower values for coupling between secondary neurons could indicate a potential weakness in our experimental paradigm. However, we can attribute these lower values to other influences. For example, secondary neurons were usually placed on opposite sides of the primary neurons (e.g., Fig. 5). This had two effects which led to weaker S/S coupling. First, secondary neurons were slightly further apart, leading to lower coupling (Fig. 11). Second, growth was nonrandom because secondary cell neurites tended to grow along existing primary cell neurites and this misrouting prevented some contacts between the growing neurites. It is noteworthy that the mean S/S coupling coefficient was 0.29 , the same as between pairs of selectively axotomized neurons 5 in ganglia (Hadley et al., 1983). Neurons in buccal ganglia also tend to grow along existing fiber tracts (unpublished observations), and perhaps higher coupling was 
prevented in both cases by the presence of preferred pathways which could have diverted part of the growth that otherwise would have made contact. Although these, or other, possible differences could have lowered S/S coupling, comparisons with the extremely low values for $\mathrm{SP} / \mathrm{S}$ coupling indicated a clear difference in competence of growing and nonyrowing neurons to form connections.

Recent experiments by Haydon et al. (1984) bear on another important aspect of this research. The present experiments do not distinguish whether the nonpermissive state of stable neurons was indeed attributable to their growth status or, alternatively, to the fact that the neurons had been in culture for 8 days at the time of contact. It is conceivable that growth had stopped by day 5-7 because the neurons were "old," and this was the reason the cells did not form electrical junctions. The experiments of Haydon et al. (1984) have demonstrated that the neurotransmitter serotonin $\left(10^{-7}\right.$ M) can selectively inhibit growth of certain neurons, even when newly plated. Using this approach, they have shown that the serotonininduced nongrowing state is associated with a nonsynaptogenic state. Moreover, after serotonin is removed, the neurons can both grow and form electrical synapses, indicating that growth states, rather than age in culture, are important in determining these neurons' capacity for electrical synaptogenesis.

Consequences of growth-dependent synaptogenesis. The formation of electrical synapses between isolated neurons in vitro has not been widely reported. Until recently, electrical synapses between mammalian neurons in vitro were seen only as rare cvents (Fischbach, 1972; Ko et al., 1976; O'Lague et al., 1978; Nurse, 1981), with chemical synaptic transmission as the primary mode of communication. Most recently, however, ready formation of abundant electrical synapses between sympathetic neurons (Higgins and Burton, 1982) and between spinal motoneurons (O'Brien et al., 1982) has been reported, under appropriate culture conditions and when neurons are not too far apart. It is possible that electrical synapses also existed in earlier experiments where chemical synaptogenesis was the primary focus of study; the electrical shunting effects of chemical synaptic interactions could have obscured electrotonic connections, especially if coupling coefficients were low (e.g., Spira and Bennett, 1971). Alternatively, electrical coupling could have escaped detection if it were transient (e.g., Bulloch and Kater, 1982; O'Brien et al., 1982). It will be of future interest to correlate growth with electrical synapse formation in other in vitro systems.

What is the possible role of growth dependence for electrical synapse formation in this system? This relationship could act to refine the connectivity possibilities for groups of neurons. For example, a first-approximation rule for establishing initial connections could be to "make a connection with anyone you contact, then decide whether that connection is appropriate." A more precise rule (which eviderilly holds for Helisoma) would more narrowly define which connections were possible, as in "if you are growing, make a connection with any growing region of a neuron, then decide whether that connection is appropriate." In this way, a particular neuron is precluded from forming connections with an entire population of cells which are not growing at the same time or locale. Thus, the requirement for conjoint outgrowth for electrical synaptogenesis can act as an intial screening mechanism and can simplify the subsequent processes of selective synapse elimination and maintenance by reducing the number of initially formed synapses.

A mechanism which determines whether an electrical synapse will or will not occur may be fundamental enough to be operant in more than a single species or phylum. Is growth involved in specific establishment of connections in other systems? Consider two examples of specific formation of electrical connections: in grasshopper embryos, pionccr motoneurons growing into the leg are guided along specific pathways by "guidepost" neurons (Bentley and Keshishian, 1982; Goodman et al., 1982; Taghert et al., 1982). When the pioneer growth cones contact the guidepost neurons in the leg, they transiently couple and perhaps exchange information before the pioneer neuron goes on to the next guidepost. Docs concurrent growth allow pioneers to couple specifically to guidepost neurons and not to other cells? This question cannot be answered from available information, although the guidepost cells do initiate growth at approximately the time of contact by the pioneer neurons. In the developing optic system of Daphnia, specific electrical coupling also occurs between growing ommatidial neurons and certain neuroblasts (Lopresti et al., 1974). The neuroblasts undergo a set of stereotyped morphological changes and wrap around the ingrowing neuron in a glial-like wrapping response. This rapid change in morphology may be related to growth in the cellular changes that occur and may be a proximal factor in the compelence to form an electrical synapse.

An obvious feature unique to growing neurons is the growth cone (Ramón y Cajal, 1928; Kater and Letourneau, 1985). Growth cones are highly specialized organelles, with newly inserted membrane components (Pfenninger et al., 1982), a constant flux of polymerizing and depolymerizing actin filaments (Letourneau, 1981), and sites of high membrane turnover (Bunge, 1973; Rees et al., 1976; Tsui et al., 1982). Perhaps growth cones have a special competence to form junctions because necessary macromolecules (Kistler and Bullivant, 1980; Raviola et al., 1980) can be easily inserted only at that site of rapidly changing membrane composition. Or perhaps the particle-free regions of the growth cone (Pfenninger and Bunge, 1974) are free to simply fuse together as in fusion of secretory granules in exocrine cells (Chandler and Heuser, 1980). In addition to having specialized endings, growing neurons have special protein synthetic patterns, with specific proteins being transported down the growing axon (Benowitz et al., 1980; Deaton et al., 1980; Skene and Willard, 1980; Griffen et al., 1981). These proteins can then be involved in either synaptogenesis or modification of connections after they have formed (Skene et al., 1982). The stabilizing effect of the microtubular cytoskeleton (e.g., Bray et al., 1978; Letourneau, 1982), once neurites have formed, might be associated with the "resistance" to forming electrical connections expressed by stable Helisoma neurons. Any of the above phenomena could be related to a neuron's competence to form new connections. In any event, whatever the specific mechanisms may be, they are very closely associated with neuronal outgrowth and are amenable to direct experimental assessment.

\section{References}

Barker, D. L., R. G. Wong, and S. B. Kater (1982) Separate factors produced by the CNS of the snail, Helisoma stimulate neurite outgrowth and choline metabolism in cultured neurons. J. Neurosci. Res. 8. 419-432.

Bennett, M. R., P. A. McGrath, and D. F. Davey (1979) The regression of synapses formed by a foreign nerve in a mature axolotl striated muscle. Brain Res. 173: 451-469.

Bennett, M. V. L. (1966) Physiology of electrotonic junctions. Ann. N. Y. Acad. Sci. 137: 509-539.

Bennett, M. V. L. (1977) Electrical transmission: A comparison with chemical transmission. In Handbook of Physiology. Sect. 1: The Nervous System. Vol. l: Cellular Biology of Neurons, E. R. Kandel, ed., pp. 357-416.

Benowitz, L. I., V. E. Sashoua, and M. G. Yoon (1980) Rapidly transported proteins in the regenerating optic nerve of goldfish. Soc. Neurosci. Abstr. 6: 388 .

Bentley, D., and H. Keshishian (1982) Pathfinding by peripheral pioneer neurons in grasshoppers. Science 218: 1082-1088.

Bray, D. (1979) Mechanical tension produced by nerve cells in tissue culture. J. Cell Sci. 37: 391-410.

Bray, D., C. Thomas, and G. Shaw (1978) Growth cone formation in cultures of sensory neurons. Proc. Natl. Acad. Sci. U. S. A. 75: 5226-5229.

Brown, M. C., R. L. Holland, and W. G. Hopkins (1981) Motor nerve sprouting. Annu. Rev. Neurosci. 4: 17-42.

Bulloch, A. G. M., and S. B. Kater (1981) Selection of a novel connection by adult molluscan neurons. Science 212: 79-81.

Bulloch, A. G. M., and S. B. Kater (1982) Neurite outgrowth and selection of novel electrical connections by adult Helisoma neurons. J. Neurophysiol. 48: 569-583.

Bulloch, A. G. M., S. B. Kater, and A. D. Murphy (1980) Connectivity changes in an isolated molluscan ganglion during in vivo culture. J. Neurobiol. 11: $531-546$

Bulloch, A. G. M., H. R. Miller, and S. B. Kater (1982) Extant synapses play 
a role in the selective stabilization of new electrical connections in the adult Helisoma nervous system. Soc. Neurosci. Abstr. 8: 435.

Bunge, M. B. (1973) Fine structure of nerve fibers and growth cones of isolated sympathetic neurons in culture. J. Cell Biol. 56: 713-725.

Chandler, D. E., and J. E. Heuser (1980) Arrest of membrane fusion events in mast cells by quick-freezing. J. Cell Biol. 86: 666-674.

Cohan, C. S., and S. B. Kater (1983) Specification of the strength of electrica connections between isolated identified neurons in vitro. Soc. Neurosci. Abstr. 9: 372.

Cotman, C. W. (1978) Neuronal Plasticity, Raven Press, New York.

Cox, R. P. (1974) Cell Communication, John Wiley \& Sons, New York.

Deaton, M., J. A. Freeman, M. Willard, and P. Skene (1980) Quantitative two dimensional gel analysis of specific protein induction during optic nerve regeneration. Soc. Neurosci. Abstr. 6: 388.

Dennis, M. J., and J. W. Yip (1978) Furmalion and elimination of foreign synapses on adult salamander muscle. J. Physiol. (Lond.) 274: 299-310.

Dixon, W. J., and F. J. Massey, Jr. (1969) Introduction to Statistical Analysis, Ed. 3, McGraw-Hill Book Co., New York.

Fischbach, G. D. (1972) Synapse formation between dissociated nerve and muscle cells in low density cell cultures. Dev. Biol. 28: 407-429.

Gilula, N. B., O. R. Reeves, and A. B. Steinbach (1972) Metabolic coupling, ionic coupling and cell contracts. Nature 235: 262-265.

Goodman, C. S., J. A. Raper, R. K. Ho, and S. Chang (1982) Pathfinding by neuronal growth cones in grasshopper embryos. In Developmental Order: Its Origin and Regulation, S. Subtelny and P. B. Green, eds., pp. 275316. Alan R. Liss, Inc., New York.

Griffen, S. W., D. L. Price, D. B. Drachman, and J. Morris (1981) Incorporation of axonally transported glycoproteins into axolemma during nerve regen eration. J. Cell Biol. 88. 205-214.

Hadley, R. D., and S. B. Kater (1982) Growing neurites are required for formation of electrotonic connections in molluscan ganglia. Soc. Neurosci. Abstr. 8: 435

Hadley, R. D., and S. B. Kater (1983) Competence to form electrical connections is restricted to growing neurites in the snail, Helisoma. J Neurosci. 3: 924-932.

Hadley, R. D., R. G. Wong, S. B. Kater, D. L. Barker, and A. G. M. Bulloch (1982) Formation of novel central and peripheral connections between molluscan central neurons in organ cultured ganglia. J. Neurobiol. 13 . $217-230$.

Hadley, R. D., S. B. Kater, and C. S. Cohan (1983) Electrical synapse formation depends upon interaction of mutually growing neurites. Science 221: $466-468$

Hall, D. C., M. V. L. Bennett, and S. B. Kater (1984) Small gap junctions found by freeze fracture in Helisoma ganglia. Soc. Neurosci. Abstr. 10 921.

Haydon, P. G., D. P. McCobb, and S. B. Kater (1984) Serotonin selectively inhibits growth cone motility and synaptogenesis of specific identified neurons. Science 226: 561-564.

Higgins, D., and H. Burton (1982) Electrotonic synapses are formed by fetal rat sympathetic neurons maintained in a chemically-defined culture medium. Neuroscience 7: 2241-2253.

Kater, S. B., and C. R. S. Kaneko (1972) An endogenously bursting neuron in the gastropod mollusc, Helisoma trivolvis: Characterization of activity in vivo. J. Comp. Physiol. 79: 1-14.

Kater, S. B., and P. C. Letourneau (1985) Biology of the Nerve Growth Cone, Alan R. Liss, Inc., New York.

Kater, S. B., C. S. Cohan, and H. R. Miller (1982) Factors affecting the morphology of identified molluscan neurons grown in cell culture. Soc. Neurosci. Abstr. 8: 691.

Kistler, J., and S. Bullivant (1980) The connexon order in isolated lens gap junctions. J. Ultrastruct. Res. 72: 27-38

Ko, C. - P., H. Burton, and R. P. Bunge (1976) Synaptic transmission between rat spinal cord explants and dissociated superior cervical ganglion neurons in tissue culture. Brain Res. 117: 461-485.

Lawrence, T. S., W. H. Beers, and N. B. Gilula (1978) Transmission of hormonal stimulation by cell-to-cell communication. Nature 272: 501-506.

Letourneau, P. C. (1981) Immunocytochemical evidence for colocalization in neurite growth cones of actin and myosin and their relationship to cellsubstratum adhesion. Dev. Biol. 85: 113-122.

Letourneau, P. C. (1982) Analysis of microtubule number and length in cytoskeleton of cultured chick sensory ncurons. J. Ncurosci. 2: 806814

Longo, F. M., G. Lundborg, M. Manthorpe, S. D. Skaper, and S. Varon (1981) In vivo model for nerve regeneration: Presence of neuronotrophic factors. Soc. Neurosci. Abstr. 7: 470

Longo, F. M., S. D. Skaper, M. Manthorpe, G. Lundborg, and S. Varon (1982) Further characterization of neuronotrophic factors accumulating in vivo within nerve-slurip containing silicone chambers. Soc. Neurosci. Abstr. 8 : 861

Lopresti, V., E. R. Macagno, and C. Levinthal (1974) Structure and development of neuronal connectıons in isogenic organisms: Transient gap junctions between growing optic axons and laminar neuroblasts. Proc. Natl. Acad. Sci. U. S. A. 71: 1098-1102.

Murphy, A. D. (1979) Regeneration of identified neurons of the snail, Helisoma trivolvis. Ph.D. thesis, University of lowa, lowa City.

Murphy, A. D., and S. B. Kater (1978) Specific reinnervation of a target organ by a pair of identified molluscan neurons. Brain Res. 156: 322-328.

Murphy, A. D., and S. B. Kater (1980) Sprouting and functional regeneration of an identified neuron in Helisoma. Brain Res. 186: 251-272.

Murphy, A. D., R. D. Hadley, and S. B. Kater (1983) Axotomy-induced parallel increases in electrical and dye coupling between identified neurons of Helisoma. J. Neurosci. 3: 1422-1429.

Nieto-Sampedro, M., E. R. Lewis, C. W. Cotman, M. Manthorpe, S. D. Skaper, G. Barbin, F. M. Longo, and S. Varon (1982) Brain injury causes a timedependent increase in neuronotrophic activity at the lesion site. Science 217: 860-861.

Nurse, C. (1981) Interactions between dissociated rat sympathetic neurons and skeletal muscle cells developing in cell culture. Dev. Biol. 88: 71-79.

O'Brien, R. J., L. W. Role, and G. D. Fischbach (1982) Characterization of identified motoneurons in vitro. Soc. Neurosci. Abstr. 8: 129.

O'Lague, P. H. E. J. Furshpan, and D. D. Potter (1978) Studies on rat sympathetic neurons developing in cell culture. II. Synaptic mechanisms. Dev. Biol. 67: 404-423.

Ptenninger, K. H., and R. P. Bunge (1974) Freeze-fracturing of nerve growth cones and young fibers. A study of developing plasma membrane. J. Cell Biol. 63: $180-196$.

Pfenninger, K. H., M. P. Johnson, and L. Ellis (1982) Growth-factor-induced phospholipid methylation in nerve growth cones. Soc. Neurosci. Abstr. 8: 927

Pitts, J. C., and J. W. Simms (1977) Permeability of junctions between animal cells. Intercellular transfer of nucleotides but not of macromolecules. Exp. Cell Res. 104: 153-163

Purves, D., and J. W. Lichtman (1980) Elimination of synapses in the developing nervous system. Science 210: 153-157

Ramón y Cajal, S. (1928) Degeneration and Regeneration of the Nervous System, R. M. May, trans!., Hafner, New York (1959).

Raviola, E., D. A. Goodenough, and G. Raviola (1980) Structure of rapidly frozen gap junctions. J. Cell Biol. 87: 273-279.

Rees, R. P. M. B. Bunge, and R. P. Bunge (1976) Morphological changes in the neuritic growth cone and target neuron during synaptic junction development in culture. J. Cell Biol. 68: 240-263.

Skene, $\mathrm{P}$., and M. Willard (1980) Two axonally transported proteins associated with growing axons in rabbits. Soc. Neurosci. Abstr. 6:687.

Skene, P., M. Willard, and J. A. Freeman (1982) Modification of an axonally transported protein in toad retinotectal terminals. Soc. Neurosci. Abstr. 8 : 865.

Slack, J. R. (1978) Interactions between foreign and regenerating axons in axoloti muscles. Brain Res. 146: 172-176.

Spira, M. E., and M. V. L. Bennett (1971) Synaptic control of electrotonic coupling between neurons. Brain Res. 37: 294-300.

Sumner, B. E. H. (1975) A quantitative analysis of the response of presynaptic boutons to postsynaptic motor neuron axotomy. Exp. Neurol. 46: 605615.

Sumncr, B. E. H. (1977) Ultrastructural responses of the hypoglossal nucleus to the presence in the tongue of botulinum toxin. A quantitative study. Exp. Brain Res. 30: 313-321.

Taghert, P. H., M. J. Bastiani, R. K. Ho, and C. S. Goodman (1982) Guidance of pioneer growth cones: Filopodial contacts and coupling revealed by an antibody to Lucifer Yellow. Dev. Biol. 94: 391-399.

Thompson, W. (1978) Reinnervation of partially denervated rat soleus muscles. Acta Physiol. Scand. 103: 81-91.

Tsui, H. T., H. Ris, and W. L. Klein (1982) High voltage electron microscopy (HVEM) of growth cones shows the presence of vesicles interconnected by a network of filaments. Soc. Neurosci. Abstr. 8: 302.

Wong, R. G., R. D. Hadley, S. B. Kater, and G. C. Hauser (1981) Neurite outgrowth in molluscan organ and cell cultures: The role of conditioning factor(s). J. Neurosci. 1: 1008-1021.

Wong, R. G., E. C. Martel, and S. B. Kater (1983a) Conditioning factor(s) produced by several molluscan species promote neurite outgrowth in cell culture. J. Exp. Biol. 105: 389-393

wong, R. G., D. L. Barker, S. B. Kater, and D. A. Bodnar (1983b) Neurite growth-promoting activity produced by the central nervous system of Helisoma. Brain Res. 292: 81-91. 